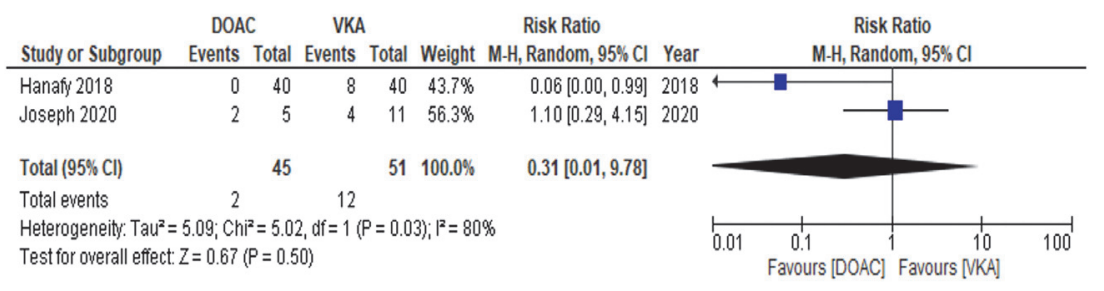

Abstract IDDF2021-ABS-0172 Figure 5 Pooled risk of death

Results From 943 citations, we included a total of 11 studies (10 observational and 1 randomized trial) evaluating 4 types of DOACs (rivaroxaban, apixaban, edoxaban and dabigatran) that fulfilled the inclusion criteria. 3 studies included patients with Child-Turcott-Pugh (CTP)-C cirrhosis. The overall pooled rate of PVT recanalization, PVT progression, major bleeding and death were $46.0 \%, 12.9 \%, 7.9 \%$ and $10.2 \%$, respectively. We found that DOACs were associated with a higher pooled rate of PVT recanalization $(\mathrm{RR}=1.67,95 \% \mathrm{CI}$ : 1.02, 2.74, $\mathrm{I} 2=79 \%)$ and lower risk of PVT progression $(\mathrm{RR}=0.14$, 95\%CI: $0.03-0.57, \mathrm{I} 2=0 \%)$. The pooled risk of major bleeding $(\mathrm{RR}=0.29,95 \% \mathrm{CI}: 0.08-1.01, \mathrm{I} 2=0 \%)$, variceal bleeding $(\mathrm{RR}=1.29,95 \% \mathrm{CI}: 0.64-2.59, \mathrm{I} 2=0 \%)$ and death $(\mathrm{RR}=0.31$, 95\%CI: $0.01-9.578, \mathrm{I} 2=80 \%)$ were similar between DOACs and VKAs. (IDDF2021-ABS-0172 Figure 2, IDDF2021-ABS0172 Figure 3, IDDF2021-ABS-0172 Figure 4)

Conclusions For the treatment of PVT in patients with cirrhosis, the bleeding risk was comparable between DOACs and VKAs. However, DOACs were associated with a higher pooled rate of PVT recanalization.

\section{IDDF2021-ABS-0184 GLOBAL INCIDENCE AND RISK FACTORS OF PANCREATIC CANCER AMONG YOUNG ADULTS: AN EPIDEMIOLOGICAL STUDY}

\begin{abstract}
${ }^{1}$ Junjie Huang*, ${ }^{1}$ Alfonse Ngai, ${ }^{2}$ Veeleah Lok, ${ }^{3}$ Xianjing Liu, ${ }^{4}$ Lin Zhang, ${ }^{5}$ Jinqiu Yuan, ${ }^{6}$ Wanghong Xu, ${ }^{7}$ Zhi-Jie Zheng, ${ }^{1}$ Martin CS Wong. ' Jockey Club School of Public Health and Primary Care, Faculty of Medicine, Chinese University of Hong Kong, Hong Kong; ${ }^{2}$ Department of Global Public Health, Karolinska Institute, Karolinska University Hospital, Stockholm, Sweden; ${ }^{3}$ Department of Radiology and Medical Informatics, Erasmus University Medical Centre, Rotterdam, Netherlands; ${ }^{4}$ Melbourne School of Population and Global Health, The University of Melbourne, Victoria, Australia; ${ }^{5}$ Clinical Research Centre, The Seventh Affiliated Hospital, Sun Yat-sen University, Shenzhen, Guangdong, China; ${ }^{6}$ School of Public Health, Fudan University, Shanghai, China; ${ }^{7}$ Department of Global Health, School of Public Health, Peking University, Beijing, China
\end{abstract}

\subsection{6/gutjnl-2021-IDDF.101}

Background The objective of this study is to examine the incidence and risk factors for pancreatic cancer among younger adults aged 15-49 years old using global and national cancer registry data.

Methods We retrieved the Global Cancer Observatory (GLO$B O C A N) 2020$ for age-standardised incidence (ASI) per 100 000 of pancreatic cancer among younger individuals from 185 countries. We estimated the prevalence of various risk factors among the younger adults for each country by joinpoint regression. We performed the multivariable linear regression to test the associations between risk factors and incidence whilst adjusting for the human development index (HDI) and gross domestic products (GDP).

Results The total number of new cases of young-onset pancreatic cancer was 27 210, with an ASI of 0.68 in 2020

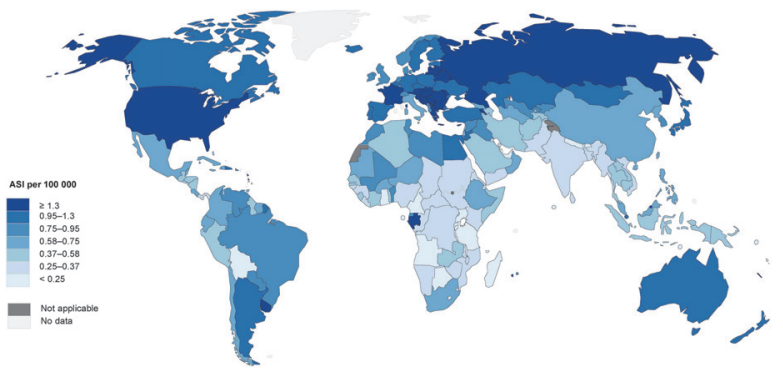

Abstract IDDF2021-ABS-0184 Figure 1 Global incidence of youngonset pancreatic cancer in 2020

(IDDF2021-ABS-0184 Figure 1. Global incidence of youngonset pancreatic cancer in 2020). The highest ASI was in males ( 0.85 vs. 0.50 in females); Central and Eastern Europe (1.4), Northern America (1.3), Western Europe (1.2); and countries of High Income (1.1) and Upper-Middle Income (0.75). Nations with a higher incidence of young-onset pancreatic cancer had a higher prevalence of tobacco use (beta coefficients $(\beta)=0.021,95 \%$ confidence intervals (CI) 0.012 0.030), alcohol consumption $(\beta=0.037$, CI 0.024-0.051), unhealthy dietary habits $(\beta=0.014$, CI $0.008-0.019)$, hypertension $(\beta=0.018$, CI 0.007-0.029), and high cholesterol $(\beta=0.019$, CI $0.011-0.026)$ among the young population.

Conclusions The burden of young-onset pancreatic cancer was notable in 2020, with higher incidence observed in male subjects and more developed regions. The associated risk factors for young-onset pancreatic cancer were tobacco use, alcohol consumption, unhealthy dietary habits, hypertension, and high cholesterol. To prevent young-onset pancreatic cancer, lifestyle modifications, metabolic diseases management, and early detection by screening among high-risk young individuals could be useful strategies.

\section{IDDF2021-ABS-0190 IMPROVING HEPATITIS B VACCINATION AND RESPONSE RATE IN LIVER TRANSPLANT RECIPIENTS}

Shirley Huey Shin Bong, Elaine Ah Gi Lo, Eunice Xiang Xuan Tan, Guan-Huei Lee. National University Hospital, Singapore

\subsection{6/gutjnl-2021-IDDF.102}

Background Hepatitis B virus (HBV) vaccination is indicated for all unexposed transplant recipients on long-term immunosuppression. However, vaccine response is often poor and monitoring overlooked. We conducted a service improvement project over five months to identify liver transplant (LT) recipients with inadequate anti-HBs titre and started them on a vaccination programme. Non-responders were identified for future intervention. 


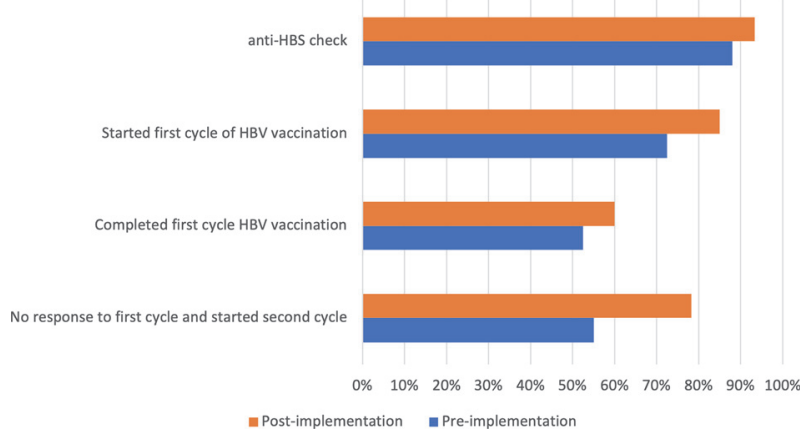

Abstract IDDF2021-ABS-0190 Figure 1

Methods A cross-sectional study of all eligible LT recipients from 2008 to 2020 in a leading transplant centre in Singapore was conducted to determine the vaccine response in HBV-naïve subjects. A standardised workflow was devised to identify barriers in vaccination and response monitoring. Transplant coordinators and pharmacists assisted physicians to identify and prescribe CDC-recommended vaccinations using a template, which include Engerix $40 \mathrm{mcg}$ of three doses, followed by rechecking anti-HBs titre one month after. A second 3 -dose Engerix regimen was given if anti-HBs remains $<10$ $\mathrm{mIU} / \mathrm{mL}$. Non-responder is defined as failure to achieve protective anti-HBs titre after completing two cycles.

Results Of the 279 LT recipients, we excluded patients not on follow-up and patients with anti-HBc positivity. 75 patients were included. Prior to vaccination implementation, 9/75 $(12 \%)$ were not checked for anti-HBs. Of the 66 checked, 40 required vaccination. $29 / 40(72.5 \%)$ were started on the first cycle, and 21/40 (52.5\%) completed. 11/20 (55\%) patients were initiated on the second cycle. Post workflow implementation, 40 needed vaccination. 34/40 (85\%) were started on the first cycle and 24/40 (60\%) completed. 18/23 (78.3\%) were started on a second cycle. 3/4 (75\%) were non-responders (IDDF2021-ABS-0190 Figure 1). Our implementation coincided with the COVID-19 pandemic where more teleconsultation was used. COVID-19 vaccination was also prioritised over HBV vaccination. We postulate that these results would be further improved once physical consultations resumed.

Conclusions The development of a standardised workflow can lead to improvement in anti-HBs testing and compliance to vaccination post-LT. These findings may be useful for other patients who are on long-term immunosuppression. Continual efforts from the multidisciplinary team are required to ensure the sustainability of effect.

\section{IDDF2021-ABS-0203 A SCREENING MODEL FOR OBSTRUCTIVE SLEEP APNEA ON THE BASIS OF FATTY LIVER DISEASE-RELATED PARAMETERS}

\footnotetext{
${ }^{1,2}$ Yang-Bor Lu*, 1,2Yu-Chieh Weng, 1,2Yung-Ning Huang, ${ }^{3}$ Hsiung-Ying Huang, ${ }^{4}$ PeiTing Cheng, ${ }^{5}$ Hui-Shan Hsieh, ${ }^{6,7}$ Ming-Shao Tsai. ${ }^{1}$ Department of Digestive Disease, Xiamen Chang Gung Hospital, Xiamen, China; ${ }^{2}$ Hepatobiliary and Pancreatic Unit, Xiamen Chang Gung Hospital, Xiamen, China; ${ }^{3}$ Department of Pulmonary and Critical Care Medicine, Sleep center, Xiamen Chang Gung Hospital, Xiamen, China; ${ }^{4}$ Formosa Biomedical Technology Corp., Taipei, Taiwan; ${ }^{5}$ Department of Otolaryngology-Head and Neck Surgery, Sleep center, Xiamen Chang Gung Hospital, Xiamen, China; ${ }^{6}$ Department of Otolaryngology-Head and Neck Surgery, Chiayi Chang Gung Memorial Hospital, Chiayi, Taiwan; ${ }^{7}$ College of Medicine, Chang Gung University, Taoyuan, Taiwan
}

10.1136/gutjnl-2021-IDDF.103
Background/Aims Obstructive sleep apnea (OSA) among patients with nonalcoholic fatty liver disease (NAFLD) has an emerging increased trend, thus noninvasive screening methods are urgently needed to screen for OSA risk in these patients. Therefore, we aimed to screen them while conducting an office-based survey of hepatic steatosis. The routine hepatic check-up methods, such as controlled attenuation parameter (CAP) and hepatic steatosis index (HSI) in patients with or without OSA, are investigated and developed the screening model to detect OSA.

Methods The medical records of all adult patients (aged $\geq 18$ years) receiving routine liver sonography examination from June 2017 to June 2020 with completed CAP, polysomnography, and HSI data in our hospital were retrospectively reviewed.

Results A total of 59 patients were included in this study. Among them, 62.7\% (37/59) and 74.6\% (44/59) (detected by HSI and CAP, respectively) had NAFLD, and 78\% (46/59) were diagnosed with OSA- based on standard in-laboratory polysomnography. Binary logistic regression models showed that sex (male, odds ratio 4.17 [95\% CI: 1.76-298.92]), body mass index (BMI) (> 24.8, odds ratio 1.42 [95\% CI: 1.09 1.86]), and HSI (> 38.3, odds ratio 1.17 [95\% CI: 1.02 1.36]) significantly screening OSA risk, in descending order of odds ratio. Multivariate analysis showed that male sex, BMI, and HSI independently screen OSA and their combination best screen for OSA risk (sensitivity $=78 \%$; specificity = $85 \%$; and positive and negative predictive values $=95 \%$ and $52 \%$, respectively; area under the curve $=0.85$ ).

Conclusions Our result suggests that HSI has better screening performance than CAP. A combination of male, BMI, and HSI proposed here provides a noninvasive and rapid screening tool for OSA risk. The model can be employed while patients receive routine hepatic check-ups in clinical practice. That can be used to efficiently screen for at-risk patients, and thus facilitate earlier detection and timely treatment intervention.

\section{Clinical Gastroenterology}

\begin{tabular}{l|l}
\hline IDDF2021-ABS-0005 & NUTRITION SUPPORT TEAM FOR \\
& INTESTINAL FAILURE PATIENTS ON \\
& PARENTERAL NUTRITION: IMPROVING \\
& MACRO-AND-MICRONUTRIENTS INTAKE
\end{tabular}

Ho Yan Terry Ting*, Kai Hong, Edmond Luk, Pui Sze, Grace Lui, Hiu Yan, Sharon Chan, Ho Kin, Ivan Mak, Kai Hong, Alessandro Leung, Yuk Ying Lam, Chi Yan Wong, Wai Yin, Sally Luk. Nutrition Support Team, North District Hospital, Hospital Authority, Hong Kong

\subsection{6/gutjnl-2021-IDDF.104}

Background A nutrition support team (NST) is a multidisciplinary team of physicians, pharmacists, nurses and dietitians, who are responsible for managing patients with complex nutritional needs. NST improves the quality of treatment, clinical outcomes and reduces costs by avoiding unnecessary treatments and simplifying the treatments used. This study reviewed the outcomes achieved after the implementation of an NST on a group of patients who were given parenteral nutrition (PN) at a local acute hospital.

Methods A total of 81 patient records were retrieved for service evaluation analysis. Among the subjects, 44 of the patients on PN who were seen by the NST from Apr 2017 - Jun 2019 were compared to the historical control of 37 